Research paper

\title{
Eight-hour versus 12 -h shifts in an ICU: Comparison of nursing responses and patient outcomes
}

\author{
Joan Webster, BA, RN, RM ${ }^{\text {a, b, c, * }}$ \\ Kerri McLeod, RN, B Nurs; M Nursing Leadership; Grad Dip Nurs (Gerontology); Grad Cert \\ (Orthopaedic Nursing) a \\ Judy O'Sullivan, RN; B Nurs; M Health Admin; Grad Cert (Intensive Care Nursing); \\ Intensive Care Cert; Coronary Care Cert ${ }^{\mathrm{a}}$ \\ Laura Bird, B. Photography ${ }^{\text {a }}$

\footnotetext{
a Royal Brisbane and Women's Hospital, Herston, QLD 4006, Australia

${ }^{\mathrm{b}}$ Nursing \& Midwifery Research Centre, School of Nursing \& Midwifery, Griffith University, Nathan, QLD, 4111 Australia
} \\ ${ }^{\mathrm{c}}$ National Centre of Research Excellence in Nursing, Griffith University, Nathan QLD 411, Australia
}

\section{A R T I C L E I N F O R M A T I O N}

\section{Article history:}

Received 5 March 2018

Received in revised form

6 August 2018

Accepted 9 August 2018

\section{Keywords:}

12-h shift

Nursing workforce

Job satisfaction

Patient safety

Intensive care nursing

\begin{abstract}
A B S T R A C T
Introduction: Controversy remains about the impact of 12 -h shift patterns on staff satisfaction and health and on patient outcomes. Consequently, the objective of the study was to investigate the effect on nurses and patients of 8-h rostering compared with 12-h rostering.

Methods: We conducted a two-phase survey. Intensive care nurses completed a purposefully designed 49-item questionnaire, which included open- and closed-ended questions. Phase 1 was conducted during 2015, while the 8-h shift pattern was in place. Data for phase 2 were collected in 2017, approximately 6 months after the trial of 12 -h shifts began. We extracted data from the hospital's adverse event register to compare patient outcomes between the two phases.

Results: A total of 152/193 (78.8\%) surveys were returned in phase 1 . In phase 2, the response rate was $114 / 188(60.6 \%)$. The proportion of nurses satisfied with the roster increased 3-fold after the introduction of 12-h shifts; risk ratios 3.36 (95\% confidence intervals 2.62 to 4.28). Communication with all levels of senior staff improved, and the number of hours of professional development leave increased with the 12$\mathrm{h}$ roster phase $1,358 \mathrm{~h}$ versus $538 \mathrm{~h}$ in phase $2(\mathrm{p}=<0.0001)$. Most respondents believed that 12 -h shifts would be beneficial for their health, and this belief was validated by official leave records; there was a reduction of 69 days for sick leave and 216 days for family leave. Adverse outcomes for patients were similar in the two periods.

Conclusion: Twelve-hour shifts are popular with ICU nurses, days lost to sick and family leave are reduced, and patient outcomes are not compromised.
\end{abstract}

๑ 2018 Australian College of Critical Care Nurses Ltd. Published by Elsevier Ltd. All rights reserved.

\section{Introduction}

An 8 1/2-hour shift, which includes a 1/2-h meal break, has been the standard working model for providing 24-h nursing care for many years. This work pattern was efficient and allowed adequate

\footnotetext{
* Corresponding author at: Royal Brisbane and Women's Hospital, Level 2, Bld 34, Butterfield St, Herston, QLD 4029, Australia. Tel.: +61 736468590.

E-mail addresses: joan.webster@health.qld.gov.au (J. Webster), kerri.McLeod@ heath.qld.gov.au (K. McLeod), catriona.booker@heath.qld.gov.au (J. O'Sullivan), laurabirdphotography01@gmail.com (L. Bird).
}

time for 'handover' to the next shift of nurses. However, over the past three decades, many hospitals, particularly in Britain and North America, have adopted a 12 -h rostering pattern. ${ }^{1}$ The change was based on a belief that there may be economic advantages in reducing the number of overlaps between shifts and number of employees required. However, studies that have tested this assumption have not used formal cost analyses, which would include all health-related costs. Instead, in these studies, costs have been limited to estimates of staff salaries; they have been small, are of low quality, and have returned contradictory results. ${ }^{2-5}$ 
The introduction of 12 -h shifts was also seen as a strategy to attract and retain nurses but, again, studies have reported conflicting results. ${ }^{2,3,6,7}$ Despite the limited and controversial organisational outcomes, a change to longer shifts was welcomed by nurses, with extended hours enabling fewer days to be worked each week. ${ }^{7,8}$ Fewer days worked provides greater flexibility and opportunities for activities, thereby improving work-life balance. $^{2,9-11}$ Moreover, as the majority of nurses are women and many are mothers, fewer working days mean that less child care is required, providing an economic incentive to support the change. ${ }^{7,9,12}$ Additionally, for those living many kilometres from their workplace, travel and parking costs may be considerably reduced. ${ }^{7,8}$

More recently, concerns have been raised about 12-h shifts in relation to quality of care ${ }^{13}$ and nurses' health. ${ }^{3,9,14}$ For example, investigators of a large study of 25,924 European nurses concluded that nurses chose to work longer shifts to improve work-life balance, even though their choice may affect patient safety and their own health. ${ }^{9}$ However, a comprehensive review that included 95 studies related to 12 -h shifts, concludes that there remains insufficient evidence to support or refute the advantages of longer shifts. ${ }^{1}$ The review found contradictory results between studies in terms of patient safety and satisfaction; the risks to nurses; their experiences; and the 'organisation of work' under a 12 -h schedule. Many of the included studies were methodologically weak, and the authors recommended that further research was required to evaluate the impact of 12 -h shifts on patients and staff. ${ }^{1}$

In Australia, 12-h shifts have generally been restricted to intensive care units, 6,10,12 with the first report appearing in $1998 .^{10}$ At our hospital and at the request of staff, some rosters included a combination of $12-, 10-$, or 8 -h shifts. While this arrangement suited staff, the roster pattern often created long changeover periods and was seen as uneconomical. Consequently, in 2012, nursing management decided to abolish 10 -h and 12-h shifts. Although the decision was unpopular, intensive care services (ICU) ceased rostering 12-h shifts in November 2012. However, reverting to an 8-h roster created social and personal difficulties for many staff members, particularly for those with child minding and other caring responsibilities. Senior nursing staff also believed that opportunities for communication and education were reduced under the 8-h roster pattern. After the decision, the service experienced an exodus of highly skilled staff to other hospitals, those that still offered a 12-h shift option.

In response, the Nursing Director, Critical Care and Clinical Support Services, sought support from hospital's Nursing and Midwifery Executive Council to explore reintroduction of flexible rostering patterns to intensive care, which would include 12-h rostering pattern. The introduction of 12 -h shift patterns also required support from the Queensland Nurses and Midwifery Union and support from the majority of the nursing staff who would be affected. So, in consultation with the union and before the reintroduction of 12 -h shifts, a decision was taken to trial the change and to formally evaluate the effect of the change by surveying nurses during 8-h rostering period and again after the roster was changed back to 12 -h shifts. Consequently, the aim of the present study was to investigate the effect on nurses and patients of 8 -h rostering compared with 12 -h rostering.

\section{Methods}

\subsection{Design}

We conducted a two-phase survey. Phase 1 was conducted during 2015, while the 8-h shift pattern was in place. Data for phase
2 were collected in 2017, approximately 6 months after the trial of 12-h shifts began.

\subsection{Staff outcomes}

Satisfaction with rosters; communication with a number of levels of senior nursing staff; number of hours of staff development education; work-life balance; and number of sick and family care leave days.

\subsection{Patient outcomes}

All adverse events; medication errors; pressure ulcers; and falls

\subsection{Population and setting}

The study hospital is a tertiary referral teaching hospital with more than 900 beds, located in South East Queensland, Australia. Critical Care and Clinical Support Services is one of the largest service lines in the hospital and provides comprehensive critical care and clinical support services; it is also a referral service for other hospitals in Queensland and northern New South Wales. In the intensive care unit, 22 beds are staffed and approximately 150 full-time equivalent nurses are employed. All registered nurses (entry-level nurse) and clinical nurses (those with a sound knowledge in a specific field of practice), who worked on the clinical roster, were invited to participate in the study.

\subsection{Procedure}

A purposefully designed 49-item questionnaire, which included open-ended and closed-ended questions, was developed collaboratively between the Nursing Director, Critical care and Clinical Support Services, and Queensland Nurses Union (questionnaire available on request). Survey questions were based on section 15.3 of the Nurses and Midwives (Queensland Health) Award-State 2015, ${ }^{15}$ which contains requirements for evaluating the introduction of increased working hours. Items were critically reviewed for readability and relevance by a researcher with expertise in questionnaire design. After the review, the questionnaire was modified in line with suggestions, but it was not piloted before administration.

The survey was endorsed by Executive Director of Nursing and Midwifery Services and the hospital's Nursing and Midwifery Executive Council. All registered nurses and clinical nurses working in intensive care services were handed a copy of the survey by the unit's Clinical Nurse Consultant or by posting the survey, along with a reply-paid envelope, to their home address. Organisationally, Clinical Nurse Consultants are not permitted to work 12-h shifts, so these nurses had no vested interest in the study outcome. Nursing staff members were informed that their decision to complete the survey was completely voluntary and confidential. To ensure anonymity, there were no identifiers on the survey form. Staff members were asked to return their completed surveys, in the envelope provided to the Nursing Directors Executive Support officer or to place it in a collection box that was provided in the clinical unit.

We also interrogated several organisational databases to quantify nursing outcomes (such as sick leave, professional development leave, and family leave) and patient outcomes (all adverse events, pressure injuries, falls, and medication errors).

\section{Sample size calculation}

We based our sample size on an assumed rate of $25 \%$ of respondents who would rate satisfaction with their roster as either 'satisfied' or 'very satisfied' in phase 1 . To increase this rate to $50 \%$, 
with an $\alpha=5 \%$ and $\beta=80 \%$ in phase 2 , we would require a sample size of approximately 110 participants in both phases of the study. This number would also be sufficient to assess any increase in levels of communication with senior staff from an assumed 30\% 'often' or 'always' response in phase 1 to $50 \%$ in phase 2 .

\section{Analysis}

Data were entered and analysed using SPSS version 23 (Armonk, NY: IBM Corp.). All data were categorical so results are reported as counts and percentages. Differences between groups for the primary outcome, satisfaction with the roster, and with the communication questions were compared using the $\chi^{2}$ statistic. Results are presented as crude risk ratios and 95\% confidence intervals. Additional analysis, such as the effect of gender; length of time in the unit; nursing grade; and employment status, on levels of satisfaction and other variables, was also calculated using the $\chi^{2}$ statistic. Qualitative data were coded and are presented as counts and percentages. Totals for qualitative responses for some items are greater than 152, and the total percent is greater than $100 \%$ because some participants provided more than one response to the question. For example, in response to the question "what are the positive aspects of the 12-h shift trial?" nurses frequently cited several issues such as work-life balance, continuity of care, or less child care required, each of which was coded separately.

Incidence per 1000 was calculated using the formula 'number of events (e.g. falls) multiplied by 1,000 , divided by the appropriate denominator' (e.g. total number of occupied bed days in the target year).

Databases for the calendar year 2013, which was approximately 12 months after the 8-h roster period was introduced, were interrogated to extract data for phase 1 . We used 2017 databases for phase 2 .

\section{Results}

\subsection{Sample}

Of the 193 surveys distributed in phase 1, a total of 152 (78.8\%) were returned. In phase 2, 188 surveys were distributed and 114 (60.6\%) returned. Consequently, 266 responses were included in the analysis. A total of 209 (78.6\%) respondents were female and 98 (36.8\%) were employed full time, which means that they were employed for more than $72 \mathrm{~h} /$ fortnight. Most respondents (205, $77.1 \%)$ were registered nurses. There were 63 (23.7\%) participants who had worked in the unit less than 12 months; 58 (21.8\%) for 1-3 years; 21 (7.9\%) for $4-5$ years, and 124 (46.6\%) who had been in ICU for more than 5 years (Table 1 ).

Table 1

Participant characteristics in phase 1 and phase 2 .

\begin{tabular}{|c|c|c|c|}
\hline Participant characteristics & $\begin{array}{l}8 \text {-h phase } 1 \\
N=152 \\
N(\%)\end{array}$ & $\begin{array}{l}12-\mathrm{h} \text { phase } 2 \\
\mathrm{~N}=114 \\
\mathrm{~N}(\%)\end{array}$ & P-value \\
\hline \multicolumn{4}{|l|}{ Employment status } \\
\hline Full time & $53(34.9)$ & 45 (39.5) & 0.26 \\
\hline \multicolumn{4}{|l|}{ Length of time in ICU } \\
\hline$\leq 12$ months & $52(34.2)$ & $11(9.6)$ & \\
\hline $1-3$ years & $19(12.5)$ & $39(34.2)$ & \\
\hline $4-5$ years & $13(8.6)$ & $8(7.0)$ & \\
\hline$>5$ years & $68(54.8)$ & $56(49.1)$ & $<0.001$ \\
\hline \multicolumn{4}{|l|}{ Position } \\
\hline Registered nurse & $119(78.3)$ & $86(75.4)$ & \\
\hline Clinical nurse & $33(21.7)$ & $28(24.6)$ & 0.34 \\
\hline
\end{tabular}

\subsection{Satisfaction}

Compared with responses to the phase 1 survey, where $29.6 \%$ of staff were either satisfied or very satisfied with their roster, satisfaction increased over 3 -fold to $99.2 \%$ after the 12 -h roster was reintroduced. Statistically higher levels of satisfaction were associated with being in the unit longer $(\mathrm{p}=<0.006)$ but not with gender or employment status.

Overall, respondents rated their communication as 'often or always' with the Clinical Nurse Consultant (63\%) and the nurse educator (57\%) more frequently than with other members of the senior management group. There was an increase between phase 1 and phase 2 in the level of communication rated as 'often or always' between respondents and all levels of senior staff (Fig. 1).

\subsection{Professional development education}

Forty nurses (15\%) stated that they had accessed professional development leave either often or always. Sixty-nine respondents (26\%) reported access to leave for professional development as either 'never' or 'rarely'; and total of 109 (41\%) nurses said that they had not applied for access to professional development leave. More nurses in phase 2 than in phase $1(31 ; 27 \%$ versus $9 ; 7 \%)$ stated that they accessed professional development leave 'often or always' ( $\mathrm{p}=$ $<0.0001$ ). The higher rate of professional development leave in phase 2 was supported by organisational data, which showed during 2013 that $358 \mathrm{~h}$ of professional development leave was accessed, compared with $538 \mathrm{~h}$ in 2017 ( $\mathrm{p}=<0.0001$ ). Access to professional development leave was not affected by gender, length of time working on the unit, or employment status.

\subsection{Effect on health}

Most respondents believed that 12-h shifts would be beneficial for their health. More people in phase 2 held this belief than those in phase 1 ( $72 \%$ versus $84 \% ; \mathrm{p}=0.005)$. Those who had worked in the unit $>12$ months compared with those who had worked in ICU $<12$ months were more likely to identify a positive health benefit $(\mathrm{p}=<0$ 0.0001 ). Full-time staff members were also more likely than parttime staff members to identify a good or very good effect on health with 12-h shifts $(\mathrm{p}=0.001)$. There was no difference in the number of nurses stating that they were likely to take a sick day or feel more tired at work between the two time periods. However, those working in the unit longer stated that they would be less likely to take a sick day because of tiredness ( $\mathrm{p}=<0.001$ ) and less likely to feel tired at work ( $p=0.002)$ compared with those who had been in the unit for a shorter time. Full-time staff were more likely than part-time staff to agree with the statement "that sick/family leave would be reduced with a 12-h roster ( $p=0.001$ )". To quantify these beliefs, we extracted data from the hospital's database which showed that fewer sick hours were taken in 2017 ( 34.3 per $1000 \mathrm{~h}$ ) compared with the number taken in 2013 (39.8 per $1000 \mathrm{~h} ; \mathrm{p}=<0.0001$ ).

\subsection{Sleep}

During phase 1, 32 (21.1\%) nurses stated that they had either 'plenty' or 'enough' sleep. This number increased significantly to $102(89.5 \%)$ during phase 2 ( $\mathrm{p}=<0.0001$ ). There were no differences in responses about sleeping hours by gender, length of time in the unit, or employment status.

\subsection{Issues with child care/dependents care}

A total of 98 (36.8\%) respondents had no children, so child care was not an issue for these people. The number of respondents 
J. Webster et al. / Australian Critical Care xxx (2018) 1-6

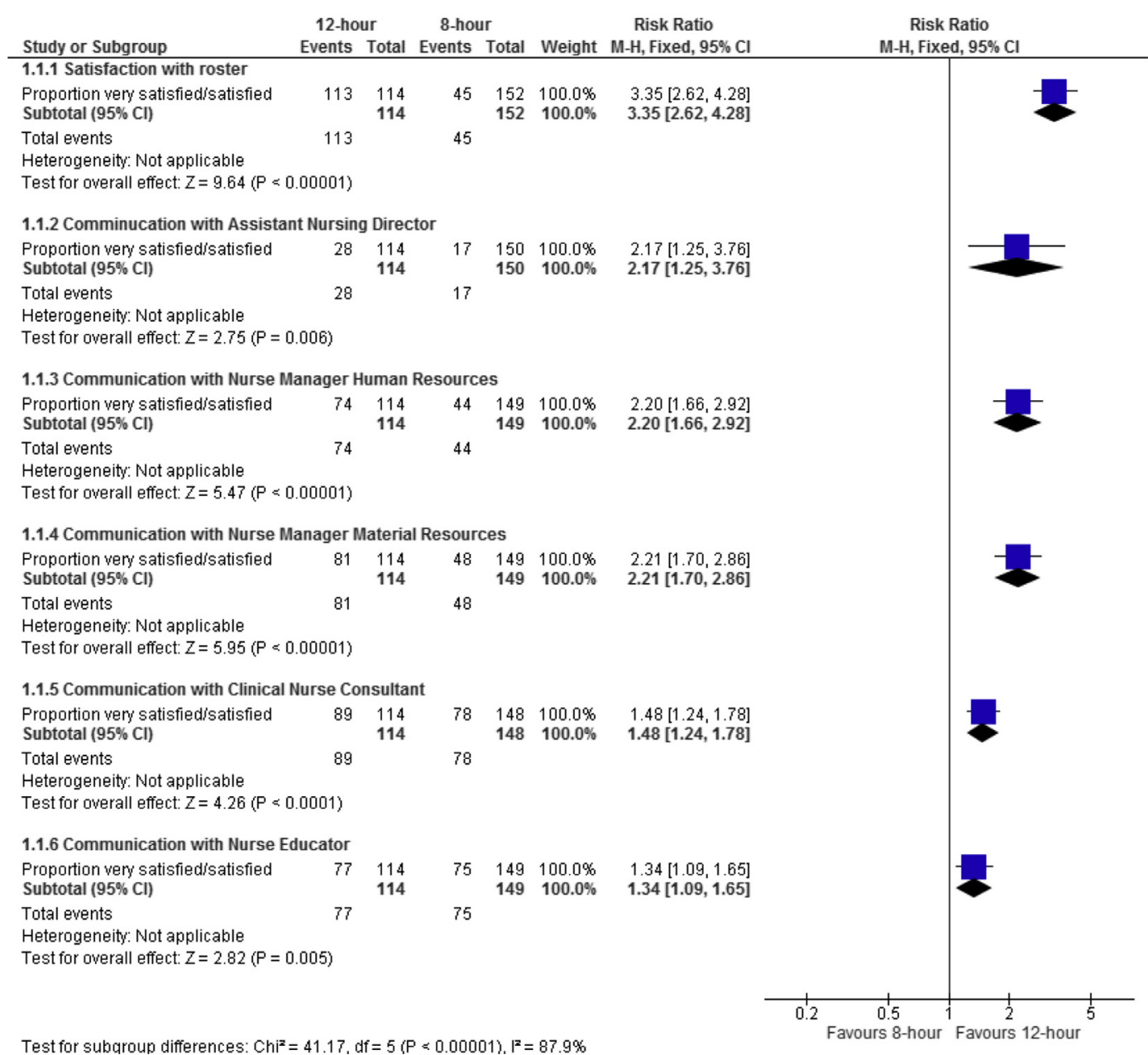

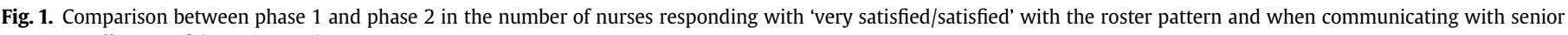
nursing staff. $\mathrm{CI}$, confidence interval.

stating they had issues related to child care reduced significantly in phase $2(\mathrm{p}=<0.001)$. There was a marginal, nonsignificant reduction in the proportion of nurses stating that they provided additional daily care to a child or adult with a disability, a medical condition, or who were frail aged from 30 (19.7\%) in phase 1 to 21 (18.4\%) in phase 2 . Nor was there a difference between phase 1 and phase 2 in relation to who cared for these people while the nurse was at work. Care was provided by their spouse/partner (8.6\%), other family members $(3.8 \%)$, a child care provider $(3.8 \%)$, or multiple different people (13.5\%). For a total of 183 (68.8\%) respondents, provision of additional daily care for a dependent was not an issue.

\section{Impact on sport/social/leisure activities}

In phase $1,120(80 \%)$ respondents stated that it was occasionally/usually or always difficult to attend sport/social/family activities. After the 12-h roster was reintroduced, the number finding it difficult to attend these activities reduced to 58 (54.2\%). The majority of respondents (86.8\%) believed that work-life balance would be improved with 12-h shifts.

\subsection{Other issues around 8- and 12-h shifts}

When asked about the benefits of the current roster, 124 (46.6\%) nurses made no comment. There were no differences in the proportion mentioning an ability to swap shifts or satisfaction with their 'mix of shifts' between the two time phases. One category (shorter shifts), which was mentioned by $46 / 63$ (73.0\%) nurses in phase 1 , was not mentioned at all in phase 2 . Two new categories emerged in phase 2 with 78/79 (98.7\%) respondents stating that work-life balance had improved with the 12-h roster and 17/79 (21.5\%) believing that there was better continuity of care.

\subsection{Work-life balance and absenteeism}

When asked specifically about work-life balance, a total of 237 (89.6\%) staff thought that work-life balance would improve with a 12-h roster. The incidence of those believing that balance would improve was no different in phase 2 (92.1\%) than in phase 1 (86.8\%). Nor was there a difference in responses to questions about the effect of 12-h shifts on absenteeism between phases. Only three (1.1\%) people overall thought absenteeism would increase. When we reviewed the official sick leave and family leave data, there was a significant reduction in both outcomes in 2017 compared with 2013. For sick leave, the reduction was 69 days and for family leave, 216 days.

\subsection{Fatigue management}

Ten $(3.8 \%)$ people thought fatigue was more easily managed with an 8-h roster compared with 216 (81\%) who thought a 12 -h roster made fatigue more manageable. More people in phase 2 
compared with phase 1 believed that fatigue would be more manageable with a 12-h roster $(p=0.023)$. Neither gender, employment status, length of time in the unit nor position influenced responses to this question. In phase 1 , more people nominated 'more days to recover' and 'less hours/less fatigue' as a reason why fatigue is more manageable with 12 -h shifts $(\mathrm{p}=0.005)$.

\subsection{Effect of 12-h roster on recruitment and retention}

Seven respondents did not answer the question. There was unanimous agreement between the remaining 259 nurses; all said that the impact of returning to 12 -h shifts would be positive. The number agreeing that there would be a positive effect did not change between phase 1 and phase 2 . Reasons why nurses believed a 12-h roster would improve retention included an 'ability to keep experienced staff; 'more flexibility'; and 'increased morale'. However, when we looked at our turnover data, we found very low rates in both time periods; 16 nurses left the unit in 2013 and 20 in 2017.

\subsection{Impact of $12-h$ shifts on patient care}

The most common response to this question was a belief that continuity of care would be improved with a 12-h roster with $81 \%$ in phase 1 and $82.5 \%$ in phase 2, believing this to be the case. Other responses included 'better handovers' and nurses 'feeling less tired'. Staff members were also asked in an open-ended question about the impact of 12 -h shifts on adverse events. Similar responses were received in both time frames with approximately $33 \%$ of respondents believing that errors would be reduced and 5\% stating that errors would increase. Table 2 shows the actual number of events, by type, in each time frame. Reported rates for all events were similar in both phases. When asked about how 12-h shifts would better meet patients' needs, there were no differences between phase 1 and phase 2 responses, with $58 \%$ of the combined sample nominating 'continuity of care'. Other responses included 'better handover' (51\%) and 'increased morale' (17\%).

\section{Discussion}

Based on self-report, staff satisfaction increased with the reintroduction of a 12-h shift pattern. This result was consistent with many reports that span a 25-year time period and include a variety of countries. $3,7,9,11,12$ Satisfaction was associated with more days off, greater flexibility in being able to organise life, and better work-life balance. In phase 1 , approximately $47 \%$ of nurses were satisfied with the 8-h shift pattern; the dramatic increase in the number being "very satisfied' or 'satisfied' with their shift after the reintroduction of 12-h shifts is probably explained by two factors. First, long-term staff members were very negative about changing from 12 -h shifts to an 8 -

Table 2

Adverse events reported to the organisation's adverse events register. Outcomes shown as number (percent) and rate per 1000 occupied bed days (OBD).

\begin{tabular}{lll}
\hline Type of event & 8-h phase 1 & 12-h phase 2 \\
\hline Medication error & $79(1.1)$ & $110(1.3)$ \\
Per OBD & 10.8 & 13.2 \\
Pressure ulcer & $235(3.2)$ & $33.1271(3.3)$ \\
Per OBD & 32.2 & 33.1 \\
Falls & $3(0.04)$ & $3(0.04)$ \\
Per OBD & 0.41 & 0.41 \\
aTotal number of incidents & $525(7.2)$ & $626(7.6)$ \\
Per OBD & 71.9 & 76.4 \\
\hline
\end{tabular}

a Total number of incidents included issues such as pathology errors, patient accidents, behavioural problems, and harm from an unknown cause and includes medication errors pressure ulcers and falls. hr roster, so were likely to have reported that they were very dissatisfied with roster in phase 1 . Second, newer staff members those who had not been previously exposed to 12 -h shifts were probably quite comfortable with a 'normal' roster pattern in phase 1. Presumably, once exposed to the advantages of a 12 -h roster, their opinion changed.

We were particularly interested in evaluating the effect of changing shift patterns on communication between various levels of nursing staff. Good communication is vital to patient safety, ${ }^{16}$ and we had read reports of poorer communication with a 12-h roster. ${ }^{11,17}$ These concerns were not realised, with results showing an increase in an ability to communicate with all levels of senior staff. The increase may be due to nurses having more time to organise their work, thus having more time to connect with their nurse leaders, attend team meetings, and participate in other unit activities. We believe that the reasons why our study results differed from others are that the two trials with negative results were small ( 36 nurses in one study and 54 in the other) and both sampled nurses employed under a 12-h roster; there was no comparison with those employed under an 8 -h schedule. ${ }^{11,17}$

The two nurse-related outcomes, which we could quantify objectively, showed that nurses' perceptions were well founded. Our data showed a significant reduction in sick and family leave in 2017 compared with the same data from 2013 when an 8-h roster pattern was in place. Similar results were found in at least three other studies that compared 12-h with shorter shift patterns. ${ }^{3,18,19}$ However, two studies conducted in Australian ICUs reported no difference in absenteeism before and after the introduction of 12-h shifts, but these were small studies and unlikely to be powered to find differences. ${ }^{6,12}$ Similarly, the belief in phase 2 that there was better access to educational opportunities was substantiated, with a rise in the actual number of hours spent on professional development compared with those available in 2013. This finding is interesting because it conflicts with results from other studies that have compared educational opportunities between longer and shorter shift schedules, all of which showed improved educational opportunities with a shorter roster.,10,11,19-21 The reason for the difference is unclear but results from most of these studies were sourced from questionnaires alone, whereas we used a database of professional development hours, which may explain the difference.

Importantly, few studies have included patient outcomes when evaluating the effect of 12-hour shifts. When we interrogated incidents reported to our adverse events register, rates were similar in both periods, suggesting that shift length had little or no effect on patient outcomes. Our adverse event rates were also much lower than rates reported from a similar population. For example, while the pressure ulcer rate from our register was just over three percent, the true rate in Queensland ICUs is closer to $11 \%{ }^{22}$ It may be that only higher stage pressure ulcers are reported on the register but the same would apply in both phases of the study.

\section{Study limitations and strengths}

The main strength of the study was that it was powered to show differences between the two study phases; it is also the largest study to investigate the effect of working hours on Australian ICU nurses. Importantly, the study also includes patient outcomes, which has been a limitation of much of the literature in this area.

Apart from the objective data drawn from organisational databases, many of the self-reported responses are based on beliefs. For example, most respondents believed that continuity of care would be improved with 12-h shifts but we did not test if this did occur. The study would have been strengthened if a matched sample had been used but, as we had decided that questionnaires would remain anonymous to maximise response rates, matching was not possible. The study was limited to one organisation, so external validity may 
be compromised. Fewer responses were received in the second phase of the survey, so response bias must be considered. Finally, objective data related to nursing outcomes, such as professional development leave, sick leave etc., were for all nurses in ICU, not just those completing surveys. However, with an overall response rate of more than $70 \%$ and given that the two time periods may have been similarly affected, we believe that our results remain valid.

\section{Conclusion}

Twelve-hour shifts are popular with ICU nurses. Satisfaction with work, communication with colleagues, educational opportunities, and quality of life is increased when longer working hours are combined with fewer days at work. In addition, days lost to sick and family leave are reduced, and, importantly, patient outcomes are not compromised.

\section{CRediT authorship contribution statement}

Joan Webster: assisted with design of methodology, applied statistical techniques to analyse data, prepared the initial and final drafts of the manuscript. Kerri McLeod: conceptualised the project, assisted with management and coordination for the project, assisted with design of methodology, contributed to reviewing and commenting on the initial and final drafts of the manuscript. Judy o'Sullivan: conceptualised the project, assisted with management and coordination for the project, assisted with design of methodology, contributed to reviewing and commenting on the initial and final drafts of the manuscript. Laura Bird: assisted with data analysis and contributed to reviewing and commenting on the initial and final drafts of the manuscript.

\section{References}

[1] Harris R, Sims S, Parr J, Davies N. Impact of $12 \mathrm{~h}$ shift patterns in nursing: a scoping review. Int J Nurs Stud 2015;52(2):605-34.

[2] Jones JJ, Brown RM. A survey of the 12-hour nursing shift in 25 North Carolina hospitals. Nurs Manag 1986;17(5):27-8.
[3] Stone PW, Du Y, Cowell R, Amsterdam N, Helfrich TA, Linn RW, et al. Comparison of nurse, system and quality patient care outcomes in 8-hour and 12 hour shifts. Med Care 2006;44(12):1099-106.

[4] Ganong WL, Ganong JM, Harrison ET. The 12-hour shift: better quality, lower cost. J Nurs Adm 1976;6(2):17-29.

[5] Wootten N. Implementing 12-hour shifts on a cardiology nursing development unit. Br J Nurs 2000;9(19):2095-9.

[6] Breeding J. The introduction of optional 12-hour shifts for nurses in an Australian Intensive Care. 2005.

[7] Houston R. Twelve-hour shifts: answer to job satisfaction? Nurs Manag $1990 ; 21(10) .88 \mathrm{~F}, \mathrm{H}$.

[8] Underwood AB. What a 12-hour shift offers. Am J Nurs 1975;75(7):1176-8.

[9] Estryn-Behar M, Van der Heijden BI, Group NS. Effects of extended work shifts on employee fatigue, health, satisfaction, work/family balance, and patient safety. Work 2012;41(Suppl 1):4283-90.

10] Campolo M, Pugh J, Thompson L, Wallace M. Pioneering the 12-hour shift in Australia-implementation and limitations. Aust Crit Care 1998;11(4): $112-5$.

[11] McGettrick KS, O'Neill MA. Critical care nurses-perceptions of 12-h shifts. Nurs Crit Care 2006:11(4):188-97.

[12] Dwyer T, Jamieson L, Moxham L, Austen D, Smith K. Evaluation of the 12hour shift trial in a regional intensive care unit. J Nurs Manag 2007;15(7): $711-20$.

[13] Ball J, Day T, Murrells T, Dall'Ora C, Rafferty AM, Griffiths P, et al. Crosssectional examination of the association between shift length and hospital nurses job satisfaction and nurse reported quality measures. BMC Nurs 2017;16:26.

[14] Stimpfel AW, Sloane DM, Aiken LH. The longer the shifts for hospital nurses, the higher the levels of burnout and patient dissatisfaction. Health Aff 2012;31(11):2501-9.

[15] Nurses and Midwives (Queensland Health) Award - State. Twelve hour shift arrangements. Part 5, 15.3. 2015. p. 32-3. http:www.qirc.qld.gov.au.

[16] Mujumdar S, Santos D. Teamwork and communication: an effective approach to patient safety. World Hosp Health Serv 2014;50(1):19-22.

[17] Ugrovics A, Wright J. 12-hour shifts: does fatigue undermine ICU nursing judgments? Nurs Manag 1990;21(1).64A, D, F-G.

[18] Lea A, Bloodworth C. Modernising the 12-hour shift. Nurs Stand 2003;17(19): 33-6.

[19] Palmer J. Eight- and 12-hour shifts: comparing nurses' behavior patterns. Nurs Manag 1991;22(9):42-4.

[20] Reid N, Todd C, Robinson G. Educational activities on wards under 12 hour shifts. Int J Nurs Stud 1991;28(1):47-54.

[21] Richardson A, Dabner N, Curtis S. Twelve-hour shift on ITU: a nursing evaluation. Nurs Crit Care 2003;8(3):103-8.

[22] Coyer F, Miles S, Gosley S, Fulbrook P, Sketcher-Baker K, Cook JL, et al. Pressure injury prevalence in intensive care versus non-intensive care patients: a statewide comparison. Aust Crit Care 2017;30(5):244-50. 